\title{
An interesting case of a twin pregnancy complicated with happy hypoxia, peripartum cardiomyopathy and haemolysis, elevated liver enzymes, low platelet count syndrome during the COVID-19 pandemic situation
}

\author{
Ramna Banerjee*, Farheen Rahman
}

Department of Obstetrics and Gynecology, Apollo Gleneagles Hospital, Kolkata, West Bengal, India

Received: 18 September 2020

Accepted: 30 October 2020

\author{
*Correspondence: \\ Dr. Ramna Banerjee, \\ E-mail: rban44@rediffmail.com
}

Copyright: () the author(s), publisher and licensee Medip Academy. This is an open-access article distributed under the terms of the Creative Commons Attribution Non-Commercial License, which permits unrestricted non-commercial use, distribution, and reproduction in any medium, provided the original work is properly cited.

\begin{abstract}
Ever since the beginning of the COVID-19 pandemic in December 2019, the focus has been on studying the varying clinical symptoms and signs of the disease. A condition which has been alarmingly occurring in quite a number of patients is a state of hypoxemia, of which the patient is blissfully unaware and does not have any symptoms of breathlessness or respiratory discomfort in spite of having low oxygen saturation levels. Hence the term coined for it"happy hypoxemia". We are reporting a case of a pregnant woman with twin pregnancy and pregnancy induced hypertension who rapidly progressed to hemolysis, elevated liver enzymes, low platelets syndrome without having proteinuria along with features of peripartum cardiomyopathy and also had low oxygen saturation levels (less than $72 \%$ ) though she did not have any complaints of dyspnea, orthopnea, hemoptysis, chest pain, cough or peripheral oedema. She was tested for COVID-19 with real-time polymerase chain reaction on 2 occasions a week apart and was found to be negative. Her recovery was also unexpectedly rapid and progressive.
\end{abstract}

Keywords: Blood pressure, Peripartum Cardiomyopathy, Hemolysis elevated liver enzymes, Low platelets

\section{INTRODUCTION}

Ever since the beginning of the COVID-19 pandemic in December 2019, the focus has been on studying the varying clinical symptoms and signs of the disease.

A condition which has been alarmingly occurring in quite a number of patients, is a state of hypoxemia, of which the patient is blissfully unaware and does not have any symptoms of breathlessness or respiratory discomfort in spite of having low oxygen saturation levels. ${ }^{1}$ Hence the term coined for it- "happy hypoxemia". 2

So far, all the studies describing this condition have found it to occur in COVID-19 patients or in patients with some underlying lung pathology. ${ }^{3}$
Having a high risk pregnancy added on top of it makes it all the more complicated especially when the patient's condition deteriorates rapidly as demonstrated by her blood parameters without the patient presenting with the corroborative symptoms.

We are reporting a case of a pregnant woman with twin pregnancy and pregnancy induced hypertension who rapidly progressed to hemolysis elevated liver enzymes (HELLP) syndrome without having proteinuria along with features of peripartum cardiomyopathy and also had low oxygen saturation levels (less than $72 \%$ ) though she did not have any complaints of dyspnea, orthopnea, hemoptysis, chest pain, cough or peripheral oedema. ${ }^{4}$ She was tested for COVID-19 with real time-polymerase chain reaction (RT PCR) on 2 occasions a week apart and was 
found to be negative. Her recovery was also unexpectedly rapid and progressive.

\section{CASE REPORT}

The patient is a 31 year old lady with dichorionic diamniotic twins, who conceived by in vitro fertilization (IVF) and was diagnosed with pregnancy induced hypertension at 32 weeks gestation. She was started on low dose oral labetalol at $100 \mathrm{mg}$ twice daily. She also had hypothyroidism and gestational diabetes controlled with metformin. During her IVF treatment 1 year back she had an electrocardiography (ECG) and echo done that was normal.

She presented in the emergency at 35 weeks 3 days gestation with complaints of severe epigastric pain and an episode of vomiting and reduced fetal movements for 1 day.

She did not have any complaints of shortness of breath, chest pain, headache, blurring of vision or fever. On examination her heart rate was $88 \mathrm{bpm}$ and baseline blood pressure (BP) was 160/90 $\mathrm{mmHg}$. Urine dipstix for protein was negative. Cardiotocography (CTG) was done which was reactive for both the babies and no uterine activity was noted.

Her blood tests which included complete blood count and liver function tests done 3 days before admission showed normal levels of both platelets and liver enzymes. Also her COVID-19 RT PCR test done a few days prior to admission was negative.

However, the blood tests repeated on the day of admission showed platelet counts of $1 \mathrm{lakh} / \mathrm{mm} 3$ and deranged liver function tests with total bilirubin $1.8 \mathrm{mg} / \mathrm{dl}$ and aspartate transaminase/alanine aminotransferase (AST/ALT) levels $427 \mathrm{U} / 1$ and $422 \mathrm{U} / 1$ respectively.

A diagnosis of hemolysis, elevated liver enzymes, low platelets (HELLP) syndrome was made based on her clinical features and blood reports and the decision to deliver the patient was taken. She had already received 2 doses of steroid (injection betamethasone) 3 days prior to admission.

In theatre her oxygen saturation level (SPO2) was found to be $72 \%$. She was administered high flow oxygen via a mask at $10 \mathrm{l} / \mathrm{min}$. Considering that the patient had no respiratory symptoms and the risks involved in intubating a pregnant patient with twin pregnancy under emergency conditions, decision was made by the anaesthetic and obstetric team to deliver her under a controlled spinal anaesthesia. Continuous pulse oximetry and serial arterial blood gas analysis was done (ABG).

She underwent emergency caesarean section which was uneventful and two healthy babies were delivered. In the recovery room her oxygen saturation level continued to remain low at $75-80 \%$ in spite of administering oxygen. She did not have any complaints and decision was made to shift her to intensive care unit (ICU) for monitoring and further management.

She was shifted to ICU where she was put on non-invasive artificial ventilation. At no point of time did she complain of any symptoms related to hypoxia. She had a chest X-ray done that suggested pleural effusion (Figure 1). Computed tomography pulmonary angiography (CTPA) was done that ruled out pulmonary embolism but showed bilateral pleural effusion, and interlobular septal thickening with patchy ground glass opacities in both lungs and bilateral basal consolidations (Figure 2). These findings raised a suspicion of COVID-19 infection. Hence a RT PCR for COVID-19 was repeated that was again found to be negative. ECG showed low left ventricular ejection fraction (LVEF) of 36\% with general wall hypokinesia implying peripartum cardiomyopathy. The troponin 1 (trop 1) levels were raised to 144. Arterial blood gas analysis showed a picture of metabolic acidosis with increased lactate levels $\left(\mathrm{pH}\right.$ 7.38, $\mathrm{PCO}_{2}$ 31.7, $\mathrm{PO}_{2}$ 46.2, lactate 2.75).

Blood tests were repeated the following day after caesarean section showed falling platelet levels of $60,000 / \mathrm{mm}^{3}$ and ALT/AST $382 \mathrm{U} / 1$ and $337 \mathrm{U} / 1$, lactate dehydrogenase (LDH) of 1448; characteristic of HELLP syndrome.

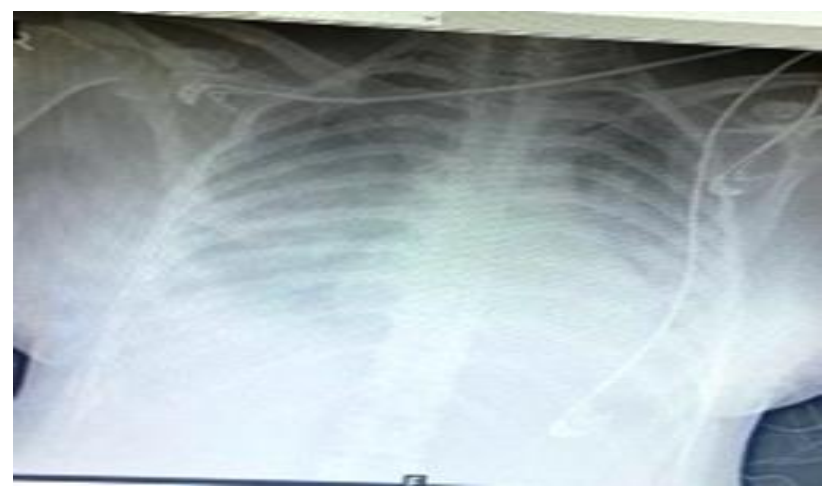

Figure 1: Pleural effusion.

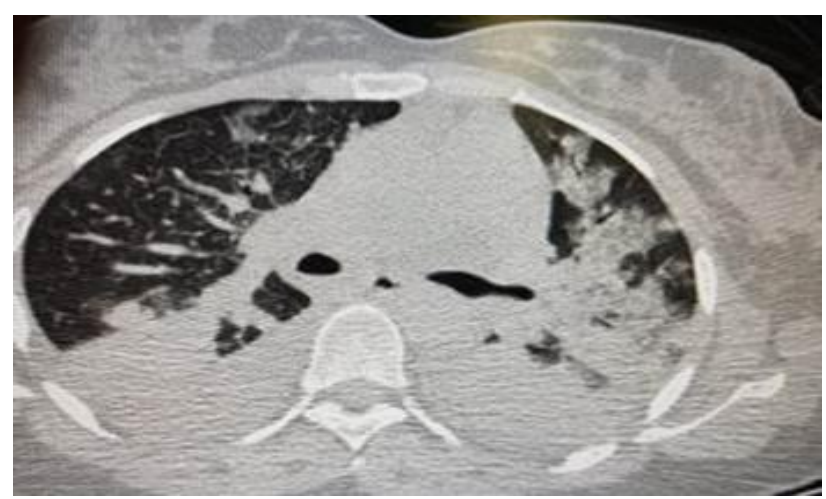

Figure 2. Inter-lobular septal thickening and bibasal consolidation. 
She was managed conservatively by a multidisciplinary team of Intensivists, anaesthetists and cardiologists in the ICU along with the obstetric team.

She was started on Intravenous antibiotics (meropenem and teicoplanin), frusemide antihypertensives (ramipril, bisoprolol, eplerenone) and thromboprophylaxis with enoxaparin. She also received prophylactic magnesium sulfate infusion $\left(\mathrm{MGSO}_{4}\right)$ that was started after delivery and continued for 24 hours. She was fluid restricted based on intake/output in 24 hours to prevent volume overload. Oral intake was allowed 24 hours after surgery.

She rapidly improved and was gradually able to maintain normal $\mathrm{O}_{2}$ saturation levels on high flow oxygen via nasal prongs only.

She was shifted to high-dependency unit (HDU) on the 3rd post-operative day and could gradually maintain normal $\mathrm{O} 2$ saturation levels on room air. Her liver function tests (LFTs) and platelets started improving and her BP remained controlled with medications.

Echocardiography was repeated 3rd day after delivery which showed left ventricle (LV) ejection fraction had marginally improved to $38 \%$.

By the 6th post-operative day she was stable enough to be shifted to the ward and platelet counts had risen to 1.6 $\mathrm{lakh} / \mathrm{mm}^{3}$ and AST and ALT were less than $100 \mathrm{U} / \mathrm{l}$, bilirubin was normal.

She was discharged 9 days after caesarean section with instructions to repeat blood tests and echocardiography after 2 weeks and follow up.

\section{DISCUSSION}

Pregnancy induced hypertension is a high risk condition as it can progress to pre-eclampsia, eclampsia and HELLP syndrome. Normally it follows a sequence of high BP followed by proteinuria progressing to the onset of convulsions and deranged blood picture. ${ }^{5}$

This patient however rapidly progressed to HELLP in a span of 4 days without having proteinuria. She also had severe hypoxemia without any symptoms of any respiratory discomfort which was also unusual.

Breathing is centrally controlled by the medulla oblongata and pons in the brainstem. The main input is from chemical feedback from central and peripheral chemoreceptors. The cerebral cortex, lungs and metabolic rate also play a role. ${ }^{6}$ When the signal of internal hypoxia is sensed by the brain the patient feels a sense of air hunger and the need to take deep breaths to counteract it. However, in patients exhibiting happy hypoxemia this sensation is absent and so they remain comfortably hypoxemic without being aware of it. The pathophysiology behind that phenomenon is still unclear and studies are still being carried out to look for an explanation. ${ }^{7}$

Until now studies have showed this low oxygen state without any symptoms seem to be present only in COVID19 infected patients or in patients with some underlying pathology like lung atelectasis, intrapulmonary shunts (arterio-venous malformations) or a right to left intracardiac shunt.

Though our patient did have low cardiac ejection fraction, there was no other pathology which could explain the severe hypoxemia. ${ }^{8}$

She also improved rapidly within a week on conservative management without the need for intubation and ventilation or prolonged stay in the ICU.

\section{CONCLUSION}

In summary, this case has shown that the state of happy hypoxemia may not be confined to COVID-19 infected patients or to patients with underlying lung pathology in which the respiratory physiology gets somehow altered leading to low $\mathrm{O}_{2}$ saturation levels without respiratory symptoms. Progesterone increases sensitivity of the respiratory centre to carbon dioxide and pregnancy especially in labour increases oxygen consumption in body significantly.

Also pregnancy induced hypertension can rapidly progress to HELLP without the intervening stage of proteinuria.

There are different opinions regarding optimal method of anesthesia for caesarean section in peripartum cardiomyopathy (PPCM). Regardless of the anesthetic technique, hemodynamic goals include avoidance of sudden variation in heart rate and blood pressure. In our case the diagnosis of PPCM was made after delivery. However a controlled regional anesthesia was deemed safe since the patient had no complaints and was continuously monitored.

Rapid recovery in cases with such sudden and quick deterioration is rare and a multidisciplinary approach with appropriate interventions, supportive management and continuous monitoring is needed for optimising outcomes. This patient was fortunate enough to recover within a span of 10 days and get discharged in a stable condition which may be considered a miracle in itself.

\section{Funding: No funding sources \\ Conflict of interest: None declared \\ Ethical approval: Not required}

\section{REFERENCES}

1. Tobin MJ, Laghi F, Jubran A. Why COVID-19 silent hypoxemia is baffling to physicians. Am J Respir Crit Care Med. 2020;202(3):356-60. 
2. Couzin-Frankel J. The mystery of the pandemic's 'happy hypoxia'. Science. 2020;368(6490):455-6.

3. Dhont S, Derom E, Braeckel EV, Depuydt P, Lambrecht BN. The pathophysiology of 'happy' hypoxemia in COVID-19. Respir Res. 2020;21(198).

4. Bhattacharyya A, Basra SS, Kar B. Peripartum Cardiomyopathy A Review. Tex Heart Inst J. 2012;39(1):8-16.

5. Weinstein L. Syndrome of hemolysis, elevated liver enzymes, and low platelet count: A severe consequence of hypertension in pregnancy. Am J Obstet Gynecol. 1982;142:159.

6. Robinson D, Gebhart F. Inside information-the unique features of visceral sensation. Mol Interv. 2008;8(5):242-53.
7. Joshi PK, Chitale MS. A comparative study of arterial oxygen saturation in pregnant and nonpregnant women. Natl J Physiol Pharm Pharmacol. 2017;7(10):1081-3.

8. Burki N, Lee L. Mechanisms of dyspnea. Chest. 2010;1385:1196-201.

Cite this article as: Banerjee R, Rahman F. An interesting case of a twin pregnancy complicated with happy hypoxia, peripartum cardiomyopathy and haemolysis, elevated liver enzymes, low platelet count syndrome during the COVID-19 pandemic situation. Int J Reprod Contracept Obstet Gynecol 2020;9:5150-3. 